\title{
La curva J nell'ipertensione. Speculazione fisiopatologica o problematica necessaria nella pratica clinica?
}

\author{
The J curve in hypertension. Physiopathologic speculation \\ or a relevant issue in clinical practice?
}

\section{Giuliano Pinna*, Claudio Pascale}

\author{
Ospedale "Cottolengo", Torino
}

Ricevuto il 12 giugno 2010; accettato il 27 settembre 2010 disponibile online il 27 novembre 2010

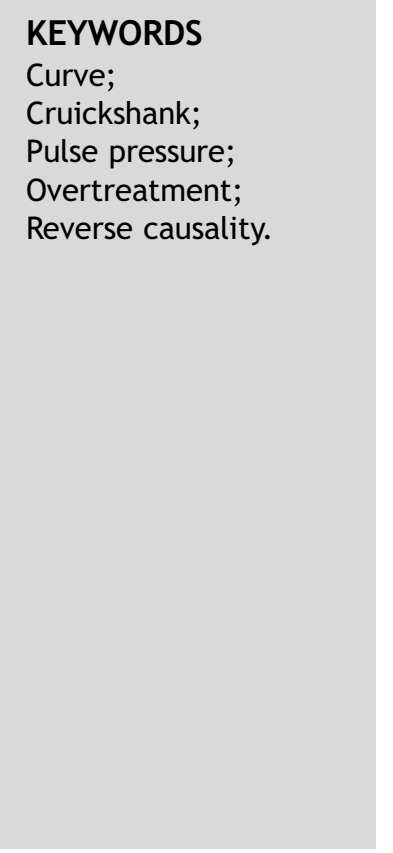

\begin{abstract}
Summary
Introduction: The J curve phenomenon in the treatment of hypertension refers to the view that reducing blood pressure below a certain critical point (diastolic pressure in particular) is no longer beneficial and may actually increase the risk of cardiovascular events. The existence of this phenomenon has been debated for many years. Many autors deny that it exists, at least within the range of pressure values commonly observed in clinical practice.

Aim of the study: The aim of this article is to verify whether in 2010 it is still possible to talk about a J curve or whether the inverse relationship between diastolic pressure and cardiovascular events depends on pre-existent diseases.

Materials and methods: The authors carried out a search of the PubMed database for articles published in 2004 or later with the following Keywords J-curve, antihypertensive treatment, and blood pressure goals.

Results: Most of the studies reviewed confirmed the existence of a J curve, but the role played by serious comorbidities is still unclear. In most cases, patients with major diseases have lower blood pressures. Moreover, cardiac patients already have impairments involving the coronary circulation. It is quite obvious that these patients are more exposed to pressure drops, especially those occurring during diastole, when the vast majority of blood flow to the coronary circulation is delivered.

Conclusions: A J curve certainly exists: there is obviously a threshold value under which an excessively low blood pressure can no longer guarantee adequate perfusion of vital organs. The problem lies in the identification of this threshold value. Furthermore, considering the undeniable impact on this phenomenon of concurrent diseases, timely intervention is essential to avoid
\end{abstract}

\footnotetext{
* Corrispondenza: via Bertola 86 - 10122 Torino.

E-mail: giuliano.pinna@gmail.com (G. Pinna).
} 
transforming low-risk patients (those less likely to experience J curve-related complications) into high-risk patients (more likely to experience these complications).

(c) 2010 Elsevier Srl. All rights reserved.

\section{Introduzione}

Studi epidemiologici di popolazione hanno evidenziato che quanto più è bassa la pressione arteriosa, tanto minore è il rischio cardiovascolare, ma nei pazienti ipertesi sottoposti a trattamento i pareri non sono unanimi. In realtà da molti anni l'esistenza di una curva $J$ nell'ipertensione fa discutere, in pratica da quando Cruickshank pubblicò un'analisi retrospettiva su 902 pazienti con ipertensione moderata/severa, riportando un aumento della mortalità in quelli con malattia coronarica (Coronary Artery Disease, CAD) se la loro pressione diastolica (PAD) veniva ridotta sotto $85-90 \mathrm{mmHg}$ [1-3].

Invero, in uno studio retrospettivo del 1979 Stewart [4] aveva segnalato che in 169 ipertesi non controllati valori di $\mathrm{PAD}<90 \mathrm{mmHg}$ erano associati a un incremento di 5 volte del rischio di infarto del miocardio (IM) rispetto a soggetti con PAD di 100-109 mmHg, ma fu il lavoro di Cruickshank et al. [3] a gettare il sasso nello stagno.

Cruickshank suddivise la PAD in terzili e riscontrò una riduzione graduale degli eventi coronarici nel terzile superiore $(<90-100 \mathrm{mmHg}$ ) fino a un beneficio massimo (nadir dell'incidenza di eventi) nel terzile intermedio, cui seguiva, nel terzile inferiore, un aumento dell'incidenza degli eventi coronarici [5] (fig. 1). Il lavoro suscitò molte polemiche, anche perché accettare l'esistenza di una curva J pone problematiche piuttosto importanti e persino rischiose, potendo ingenerare la falsa convinzione che sia dannoso abbassare la PAD in un range frequentemente incontrato nella pratica clinica. Da allora sono stati pubblicati numerosi studi con conclusioni discordanti, probabilmente perché si

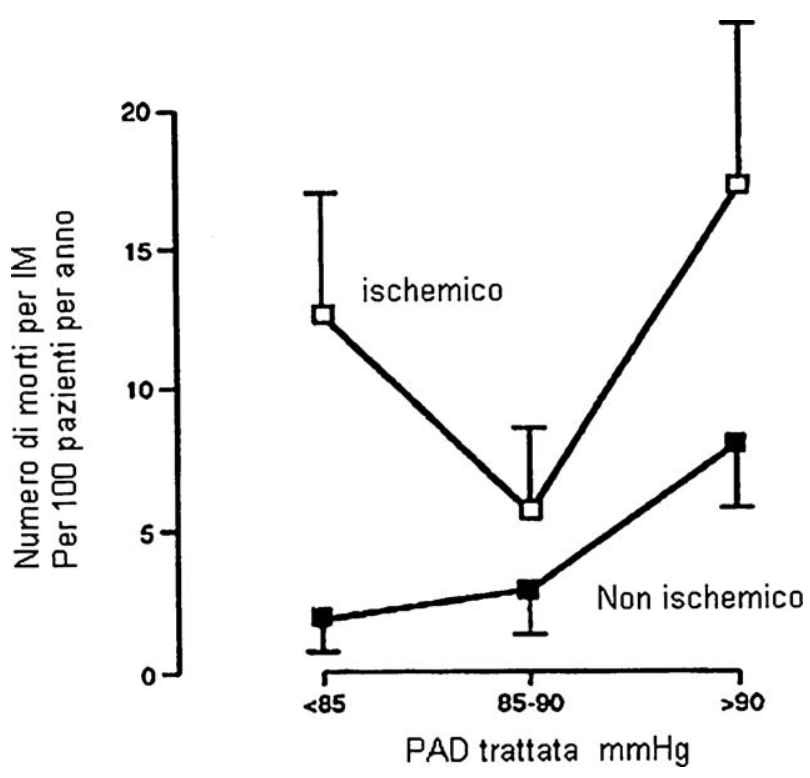

Figura 1 Fenomeno della curva J nei soggetti ischemici. Nei soggetti non ischemici il fenomeno è molto meno pronunciato. Fonte: Cruickshank JM. Cardiovasc Drugs Ther 2000;14(4):373-9. sono confrontate popolazioni di ipertesi diverse (adulti vs anziani, ipertensione sistodiastolica vs ipertensione sistolica isolata ecc.) o con protocolli diversi (differenti obiettivi pressori da raggiungere, trattamento attivo vs placebo 0 non trattamento, durata del follow-up ecc.) [6].

\section{Considerazioni fisiopatologiche}

La parte di gran lunga più importante del flusso coronarico nel ventricolo sinistro (VS) si svolge in diastole. In sistole la contrazione del VS comprime i vasi intramurali e impedisce completamente il flusso; al picco della sistole si verifica anche un flusso retrogrado nelle arterie coronarie, particolarmente nelle piccole arterie epicardiche e intramurarie.

Quando la pressione di perfusione coronarica scende sotto i $40-50 \mathrm{mmHg}$, la cosiddetta pressione a flusso zero, il flusso diastolico nelle coronarie cessa.

Inoltre, la densità capillare è ridotta in presenza di ipertrofia ventricolare sinistra (IVS), condizione tipica dell'ipertensione di lunga durata; ciò restringe il range dell'autoregolazione coronarica, specialmente nel subendocardio [7] ( fig. 2).

Se poi coesiste una CAD, un repentino abbassamento della pressione, in particolare diastolica, si può tradurre in un'ischemia silente o sintomatica in relazione temporale con l'ipotensione.

\section{Studi clinici}

Nonostante le polemiche suscitate, nel complesso gli studi clinici seguiti alla pubblicazione di Cruickshank hanno accettato una curva J soprattutto per la PAD e in particolare nei casi in cui preesisteva una CAD $[8,9]$. Tuttavia nei primi anni 2000 si affermò il concetto di "lower is better", per il quale molto influì un'ampia metanalisi di Lewington et al. [10] (1 milione di adulti in 64 studi prospettici) che affermava che la pressione arteriosa usuale è strettamente e direttamente correlata alla mortalità cardiovascolare e totale senza evidenza di una soglia, almeno fino a 115/75 $\mathrm{mmHg}$.

Studi pubblicati successivamente hanno rimesso in discussione un eccessivo abbassamento dei valori pressori. Nel 2003 venne pubblicato lo studio INVEST (The International Verapamil-Trandolapril Study) [11], in cui si osservò che il rischio di morte per tutte le cause e IM non fatale (outcome primario) raddoppiava quando la $\mathrm{PAD}$ raggiunta era $<70 \mathrm{mmHg}$ e quadruplicava quando la PAD era $<60 \mathrm{mmHg}$, con un nadir per la PAD a $84 \mathrm{mmHg}$. Questo, però, non si verificava per l'altro outcome primario: l'ictus ( fig. 3).

Lo studio INVEST dovrebbe costituire un modello ideale per l'analisi e il significato della curva J, poiché tutti i pazienti erano portatori di cardiopatia ischemica e di ipertensione. Inoltre la pressione veniva abbassata a livelli non precedentemente ottenuti e i farmaci di base utilizzati erano il verapamil e l'atenololo, ciascuno dei quali noto per ridurre 


\section{Densità capillare funzionale}

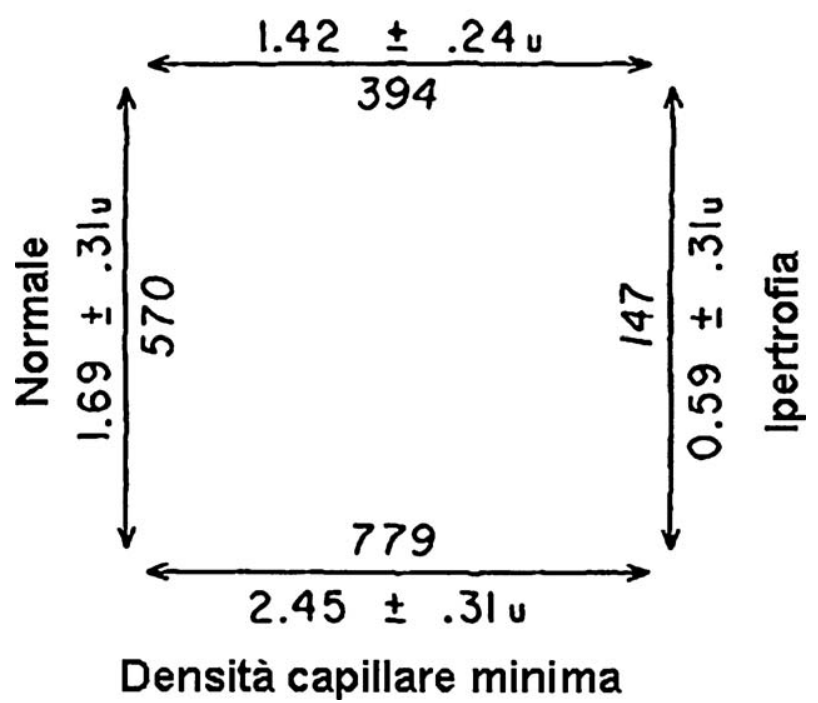

Figura 2 Densità capillare nell'animale normale e in presenza di ipertrofia ventricolare sinistra.

Fonte: modificata da Harrison DG, et al. Circulation 1985;71(6): 1135-45.

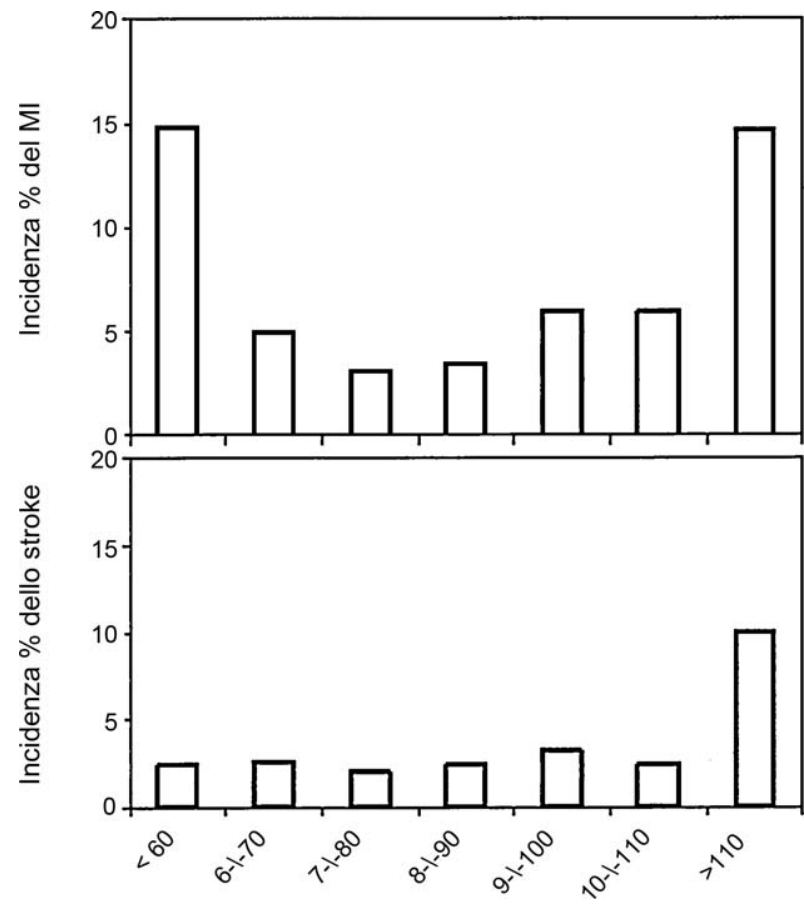

Figura 3 Incidenza di infarto del miocardio e ictus stratificata secondo la PAD nello studio INVEST.

Fonte: Messerli FH, et al. J Am Coll Cardiol 2009;54(20):1827-34.

il reinfarto. I risultati dello studio INVEST, quindi, indicano che abbassare la pressione in pazienti con CAD mediante un regime terapeutico a base di verapamil o atenololo aumenta progressivamente il rischio di IM se la PAD viene ridotta sotto $84 \mathrm{mmHg}$ e tale limite potrebbe essere più alto con farmaci meno cardioprotettivi del verapamil e dell'atenololo.
Questi risultati sono stati sostanzialmente confermati in un'analisi post hoc di vari trial, in cui è stata studiata l'associazione tra PAS, PAD e vari endpoint cardiovascolari (CV): una curva J esiste per la PAD nei pazienti con CAD e IVS, anziani e comunque ad alto rischio, ma la sua esistenza non è convincente per quanto riguarda la prevenzione dell'ictus e della malattia renale $[12,13]$.

Recentemente alcuni autori sono stati critici su tali conclusioni, rimarcando il fatto che si parla di curva J con la terapia, ma nei trial non si tiene conto dei valori di partenza della PAD, per cui è assai probabile che soggetti con bassi valori di partenza siano di base a maggior rischio di complicazioni CV (diabetici e anziani, per esempio, oltre ai cardiopatici, tutti soggetti che ovviamente hanno outcome più gravi) [14,15].

Risultati controversi si sono ottenuti in altri due ampi studi randomizzati, in doppio cieco, controllati verso placebo: lo SHEP (Systolic Hypertension in the Elderly Program) e il SystEur (The Systolic Hypertension in Europe) [16,17]. Entrambi hanno evidenziato una riduzione dell'ictus mortale e non mortale in una popolazione di anziani ipertesi trattati con farmaci attivi rispetto a quelli trattati con placebo: in pratica non è stato riscontrato un punto J. Tuttavia nel follow-up le cose sono andate diversamente: nello SHEP si è avuto un aumento di eventi in relazione all'abbassamento della PAD di $76,5 \pm 9,7 \mathrm{mmHg}$ rispetto al valore basale nel gruppo trattato con farmaci attivi, mentre nel gruppo placebo vi sono stati una riduzione dell'ictus e nessun effetto sulla cardiopatia ischemica e gli eventi CV maggiori. Nel Syst-Eur, invece, una riduzione della PAD di $85,5 \pm 5,8 \mathrm{mmHg}$ rispetto al valore basale si accompagnava in entrambi i gruppi a un aumento degli eventi non CV, mentre gli eventi CV aumentavano solo se preesisteva una cardiopatia ischemica.

Le discordanze sono da attribuire, probabilmente, alle differenze tra $i$ due studi: differivano sia $i$ valori basali sia $i$ valori più bassi di PAD ottenuti e gli schemi terapeutici (l'idroclorotiazide e il clortalidone non sono comparabili, se non altro per le proprietà farmacocinetiche assai eterogenee), ma in ultima analisi entrambi non hanno escluso riserve su un eccessivo abbassamento della PAD e si possono considerare in linea con le affermazioni di Cruickshank [3,5].

Nello studio TNT (Treating to New Targets) [18], in un gruppo di pazienti con CAD, nonostante la sostanziale riduzione dei livelli di colesterolo LDL con dosi aggressive di atorvastatina, si è riscontrata una curva $\mathrm{J}$ sia per la PAS sia per la PAD in rapporto a eventi CV, con aumento del grado di rischio ai livelli inferiori della pressione sanguigna.

Simili risultati si sono visti in un altro studio [19], e nello studio EWPHE (European Working Party on High Blood Pressure in the Elderly; idroclorotiazide-amiloride vs placebo), il trattamento attivo ha ridotto l'endpoint primario (mortalità CV ed eventi CV combinati in una popolazione anziana con ipertensione sistodiastolica), ma in una sua analisi "a posteriori" dopo 9 mesi di trattamento [20] sono state osservate, nel gruppo trattato, una relazione a $U$ tra mortalità (totale, CV e non CV) e PAS e una relazione inversa con i valori diastolici raggiunti (minore PAD, maggiore mortalità). Nel gruppo placebo, invece, la PAS aveva un rapporto lineare con la mortalità (minore PAS, minore mortalità), mentre la PAD aveva un andamento tipo J. Queste relazioni erano indipendenti da una patologia aterosclerotica preesistente, a differenza che nello studio di Cruickshank. 
I risultati di questi ultimi studi starebbero a significare che:

- esiste una curva J anche per la PAS (si veda oltre);

- la terapia (o, almeno, un eccesso di terapia) può avere un effetto negativo sulla prognosi cardiovascolare.

È evidente l'estrema cautela con la quale vanno interpretati questi dati, che quantità di registrazioni statistiche multivariate non possono scavalcare: la tentazione potrebbe essere di non trattare pazienti con PAS e pressione differenziale elevate (quindi a maggior rischio) per non abbassare ulteriormente la PAD, comportamento che può provocare danni irreparabili $[21,22]$.

\section{Studi critici verso la curva J}

Come già accennato, non esiste un accordo unanime sull'esistenza della curva J. Lo studio INVEST [11], pur favorevole, almeno per la cardiopatia ischemica, mostra che in pazienti ipertesi con CAD l'entità della pressione differenziale (Pulse Pressure, PP) durante il trattamento è un elemento predittivo degli outcome cardiovascolari più debole rispetto alla PAS, alla PAD o alla pressione arteriosa media (PAM) [23].

Inoltre, un'ampia metanalisi di Wang et al. [24] di 8 trial vs placebo su 3 popolazioni di diverse età (30-49 anni, 60-79 anni, $\geq 80$ anni) è arrivata alla conclusione che $i$ migliori risultati si ottengono con l'abbassamento della PAD anche a valori $<70 \mathrm{mmHg}$, indipendentemente dalla PAS raggiunta e dall'età. In pratica non sono state riscontrate differenze negli eventi tra giovani e anziani, anche se bisogna riconoscere che un follow-up di 4-5 anni può essere troppo breve per i giovani e troppo lungo per $\mathrm{i}$ grandi anziani.

Lo studio HOT (Hypertension Optimal Treatment) [25], dal canto suo, ha rilevato il nadir per l'endpoint primario a 138 / $85 \mathrm{mmHg}$ senza un'evidente tendenza a un aumento degli eventi CV per valori sistolici e/o diastolici $<120 / 70 \mathrm{mmHg}$, anche se si è notato un leggero incremento del rischio di mortalità CV a livelli ancora più bassi di PAD. Quindi, pur non negando il concetto della curva J, al tempo stesso lo studio HOT non suggerisce un aumento del rischio CV nei pazienti randomizzati al target più basso $(<80 \mathrm{mmHg})$ e un beneficio nei pazienti ad alto rischio.

Nel Cardiovascular Health Study, Psaty et al. [26] hanno sostenuto che l'associazione tra livelli di pressione e rischio di malattia CV era in genere lineare: in pratica non c'era evidenza di una curva J. Tuttavia, a un esame più accurato è stata osservata una relazione tipo $\mathrm{J}$ tra PAD e IM e rischio di morte con un nadir $<69 \mathrm{mmHg}$.

Nelle tabelle 1 e 2 sono elencati gli studi favorevoli alla curva J e quelli, inizialmente sfavorevoli, che l'hanno riscontrata dopo una revisione critica.

\section{Possibili meccanismi della curva J}

Si è accennato alle basi fisiopatologiche, ma quali sarebbero i meccanismi pratici con i quali si verifica la curva J? Sono state proposte tre ipotesi:

- PAD come epifenomeno di una sottostante malattia che porta indipendentemente a un aumento della morbilità/ mortalità (reverse causality);
- aumento della PP, che può riflettere una malattia vascolare avanzata e una rigidità arteriosa;

- un trattamento troppo aggressivo con ipoperfusione coronarica.

\section{Reverse causality}

Malattie croniche come neoplasie, infezioni croniche, malnutrizione e scompenso possono provocare una bassa pressione [27-30] e potrebbero essere la vera causa dell'aumento degli eventi, invece della terapia antipertensiva.

Lo studio EPESE (Established Populations for Epidemiologic Studies of the Elderly), sponsorizzato dal National Institute on Aging, ha esaminato oltre 10.000 pazienti anziani nell'arco di 5 anni per analizzare la relazione tra pressione arteriosa e cause specifiche di mortalità: a 2 anni, se la PAD era $<75 \mathrm{mmHg}$, la mortalità per tutte le cause, malattia cardiovascolare e cancro era più elevata, inducendo quindi a ritenere che ipotensione e malattie croniche siano fattori tra loro confondenti [31].

L'ipotesi che la preesistenza di malattie importanti possa influenzare l'esistenza di una curva J è sostenuta, per la PAD e per la PAS, da una metanalisi di Boutitie et al. di 7 studi randomizzati con 40.000 pazienti [32] e per la PAS dal National Health and Nutrition Examination Survey (NHANES) [33].

In contrasto, nello studio INVEST [11] né l'indice di massa corporea né la diagnosi di cancro interagiscono tra la curva J diastolica e gli outcome primari, inducendo a pensare che la perdita di peso/cachessia o le neoplasie maligne non possano essere la causa di questa condizione.

Quindi, nonostante il ruolo di epifenomeno nel determinare la curva J non possa essere escluso, l'evidenza che la $\mathrm{PAD}$ ne sia la sola o la maggiore responsabile non è convincente.

\section{Aumento della pressione differenziale}

L'aumento della PP può riflettere una malattia vascolare avanzata e una rigidità arteriosa: l'ipotesi che un aumento della PP possa essere un fattore di rischio indipendente, disgiunto dalla PAS e quindi in rapporto con la PAD, è documentata da numerosi studi [34-39], ma se ciò può essere considerato valido per la cardiopatia ischemica non sembra confermato per l'ictus: il già citato studio INVEST [11] conferma che a basse pressioni diastoliche vi è una preponderanza di infarti del miocardio rispetto agli ictus (fig. 3), anche se per quanto riguarda la PP la predittività degli eventi è più debole rispetto alla PAS, alla PAD o alla PAM.

\section{Trattamento troppo aggressivo}

Come già rilevato, un trattamento troppo aggressivo potrebbe provocare un'ipoperfusione coronarica. Sono già stati citati vari studi che mostrano una relazione tipo J per la PAD (meno chiari sono $i$ dati per la PAS) in particolare in relazione alla CAD e a uno stato preesistente di cardiopatia ischemica $[17,18]$. Sull'ictus, come si è detto, i dati sono meno convincenti e sembrerebbe addirittura non riscontrabile una curva J, neppure per la PAD. Inoltre, i pazienti 
Tabella 1 Sintesi degli studi clinici favorevoli alla curva J.

\begin{tabular}{|c|c|c|c|c|c|c|c|c|c|}
\hline Autore & $\begin{array}{l}\text { Anno } \\
\text { dello } \\
\text { studio }\end{array}$ & N. pazienti & $\begin{array}{c}\text { Età } \\
\text { (anni) }\end{array}$ & $\begin{array}{l}\text { PAD media } \\
\text { all'ingresso } \\
\text { (mmHg) }\end{array}$ & $\begin{array}{c}\text { Include soggetti } \\
\text { con malattia } \\
\text { coronarica }\end{array}$ & Infarto & Ictus & $\begin{array}{l}\text { Mortalità } \\
\text { totale }\end{array}$ & $\begin{array}{l}\text { Punto J PAD } \\
\text { (mmHg) }\end{array}$ \\
\hline Cruickshank & 1987 & 902 & 55 & 109 & Sì & Sì & No & No & $\begin{array}{l}\text { 85-90 solo pazienti } \\
\text { ischemici }\end{array}$ \\
\hline Fletcher & 1988 & 2.145 & 51 & 107 & Sì & Sì & No & No & $86-91$ \\
\hline Abernethy & 1986 & 10.053 & 51 & $90-104$ & Sì & - & - & Sì & $26^{*}$ \\
\hline Waller & 1988 & 3.350 & 50 & 110 & Sì & Sì & No & No & $91-98$ \\
\hline Coope & 1986 & 884 & 68 & 98 & Sì & Sì & - & - & $80-89$ \\
\hline Stewart & 1979 & 169 & 44 & 124 & No & Sì & - & - & $100-109$ \\
\hline Alderman & 1989 & 1.765 & 51 & 102 & Sì & Sì & No & - & $84-88$ \\
\hline Staessen & 1989 & 840 & 71 & 101 & Sì & Sì & Sì & Sì & $90-95$ \\
\hline IPPPSH & 1985 & 6,357 & 52 & 108 & No & Sì & - & - & 92 \\
\hline ANBP & 1981 & 3.931 & 50 & 101 & No & Sì & Sì & - & $85-89$ \\
\hline Wilhelmsen & 1987 & 6.569 & $40-60$ & 107 & No & Sì & Sì & Sì & $86-89$ \\
\hline Samuelsson & 1990 & 686 & 52 & 106 & Sì & Sì & Sì & - & 81 \\
\hline McCloskey & 1992 & 912 & $30-79$ & 104 & Sì & Sì & No & - & 84 \\
\hline Lindblad & 1994 & 2.574 & 59 & 92 & Sì & Sì & - & - & $90-95$ \\
\hline Somes & 1999 & 4.736 & 72 & 77 & Sì & Sì & Sì & - & $60-65$ \\
\hline Hasebe & 2002 & 234 & 64 & 88 & Sì & Sì & - & - & $95-104$ \\
\hline Pastor-Barriuso & 2003 & 783 & 54 & 82 & No & Sì & Sì & - & 80 \\
\hline Zanchetti & 2003 & 1.879 & 62 & $100-115$ & No & Sì & Sì & Sì (fumatori) & $80-85$ \\
\hline Pepine & 2003 & 22.576 & 66 & 86 & Sì & Sì & Sì & Sì & $76,4-85,8$ \\
\hline Kannel & 2004 & 7.798 & $35-80$ & - & No & Sì & Sì & No & $80-89$ \\
\hline Lubsen & 2005 & 7.661 & 63 & 80 & Sì & Sì & Sì & Sì & - \\
\hline Protegoru & 2007 & 331 & 85 & - & Sì & Sì & Sì & Sì & $<70$ \\
\hline Fagard & 2007 & 4.695 & 70 & 85 & Sì & Sì & Sì & Sì & $70-75^{*}$ \\
\hline
\end{tabular}

Fonte: modificata da Messerli FH, et al. J Am Coll Cardiol 2009;54(20):1827-34.

rivascolarizzati tollerano meglio pressioni diastoliche più basse.

Nel complesso, una netta relazione tra terapia antipertensiva e curva J non è stata ancora stabilita, anche se non tutti gli antiipertensivi hanno effetti simili:

- antagonisti del recettore dell'angiotensina, diuretici e calcio-antagonisti (CCB) migliorano la compliance arteriosa, diminuiscono maggiormente la PAS rispetto alla PAD e, quindi, riducono la PP;

- i beta-bloccanti riducono la frequenza cardiaca, aumentano lo stroke volume e hanno effetti meno favorevoli sulla $\mathrm{PP}$, ma, come i CCB non diidropiridinici (verapamil e diltiazem) permettono una perfusione diastolica più prolungata del letto coronarico;

- i farmaci che aumentano la frequenza cardiaca, come i CCB diidropiridinici a breve durata d'azione e i vasodilatatori (idralazina e minoxidil), sono più inclini a causare ischemia miocardica in soggetti suscettibili;

- i farmaci che riducono l'IVS (bloccanti il RAS, CCB ad azione prolungata) sono più efficaci nel migliorare la perfusione coronarica e la compliance arteriosa rispetto ai betabloccanti.

\section{Curva J e pressione arteriosa sistolica}

Come già accennato, alcuni studi e la metanalisi di Boutitie et al. [32] hanno sostenuto l'esistenza di una curva J anche per la PAS, almeno per pazienti con una preesistente malattia importante: la metanalisi di Boutitie et al. ha infatti riscontrato una relazione curvilinea fra PAS e mortalità totale (per trattamento attivo e controlli), mortalità CV (per i controlli) e mortalità non CV per il trattamento attivo.

Tabella 2 Studi critici sulla curva J, che l'hanno riscontrata dopo una revisione.

\begin{tabular}{|c|c|c|c|c|c|c|c|c|c|}
\hline Autore & $\begin{array}{l}\text { Anno } \\
\text { dello } \\
\text { studio }\end{array}$ & N. pazienti & $\begin{array}{l}\text { Età } \\
\text { (anni) }\end{array}$ & $\begin{array}{l}\text { PAD media } \\
\text { all'ingresso } \\
(\mathrm{mmHg})\end{array}$ & $\begin{array}{l}\text { Include soggetti } \\
\text { con malattia } \\
\text { coronarica }\end{array}$ & Infarto & Ictus & $\begin{array}{l}\text { Mortalità } \\
\text { totale }\end{array}$ & $\begin{array}{c}\text { Punto J PAD } \\
\text { (mmHg) }\end{array}$ \\
\hline Hansson & 1998 & 18.790 & 62 & 105 & Sì & Sì & No & No & $80-85$ \\
\hline Psaty & 2001 & 4.902 & 72.6 & 71 & No & Sì & No & No & $<69$ \\
\hline Glynn/PHS & 2002 & 22.071 & 53,2 & 78,8 & No & Sì & No & No & $65-70$ \\
\hline Glynn/WHS & 2002 & 39.876 & 53,8 & 77,7 & No & Sì & No & No & 70 \\
\hline
\end{tabular}

Fonte: modificata da Messerli FH, et al. J Am Coll Cardiol 2009;54(20):1827-34. 
Il già citato EPESE a 2 anni ha mostrato una curva $\mathrm{J}$ in relazione a tutte le cause di mortalità per valori $<130 \mathrm{mmHg}$ e lo studio INVEST poneva il nadir a $129 \mathrm{mmHg}$; con valori più bassi si aveva una tendenza alla curva J ( fig. 4).

Nello studio ONTARGET (Ongoing Telmisartan Alone and in combination with Ramipril Global Endpoint Trial) [40] non si è riscontrata relazione tra la riduzione della PAS e il rischio di IM, scompenso cardiaco e mortalità CV, ma si è evidenziata una curva J con un nadir intorno a una PAS di $130 \mathrm{mmHg}$ per tutti gli eventi a eccezione dell'ictus. Questa relazione tipo J non sembra avere natura causale e non deve portare a conclusioni devianti, ma è evidente che pazienti con una malattia vascolare estesa, come quelli arruolati nello studio ONTARGET, possono avere un aumentato rischio se i valori di PAS vengono abbassati sotto livelli critici.

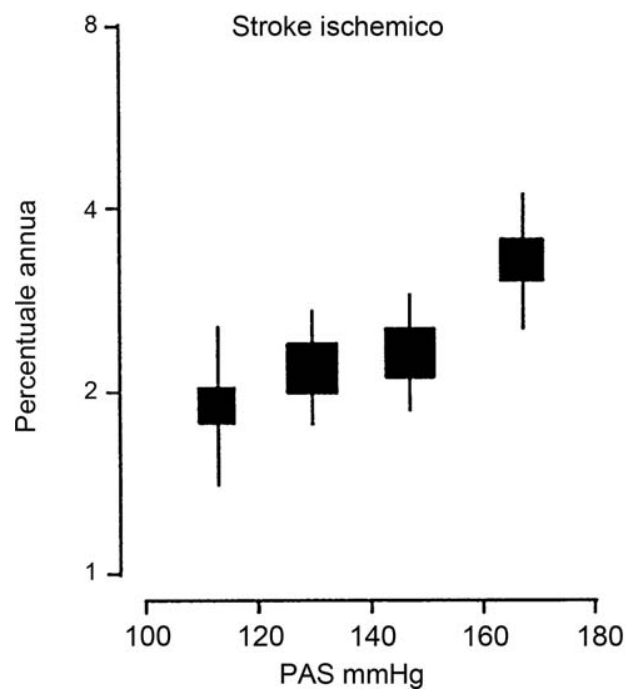

Del resto, però, lo studio PROGRESS non ha fornito un'evidenza di una curva $\mathrm{J}$ tra $\mathrm{i}$ livelli di PAS e ictus nei pazienti con malattia ischemica cerebrale e non ha identificato pazienti nei quali un più intensivo abbassamento della pressione producesse un maggiore rischio. Anzi, una PAS di $115 \mathrm{mmHg}$ era associata a una minore recidiva di ictus [41]. Ciò vale sia per l'ictus ischemico sia per quello emorragico (fig. 5).

In conclusione, la curva J per la PAS non stabilisce una relazione causale con l'endpoint primario né permette di concludere che un inappropriato decremento della pressione con la terapia causi un'eccessiva morbilità e mortalità, tuttavia occorrono maggiori evidenze a favore di un trattamento aggressivo con valori di PAS $<130 \mathrm{mmHg}$ e forse anche per valori di $130-150 \mathrm{mmHg}$.

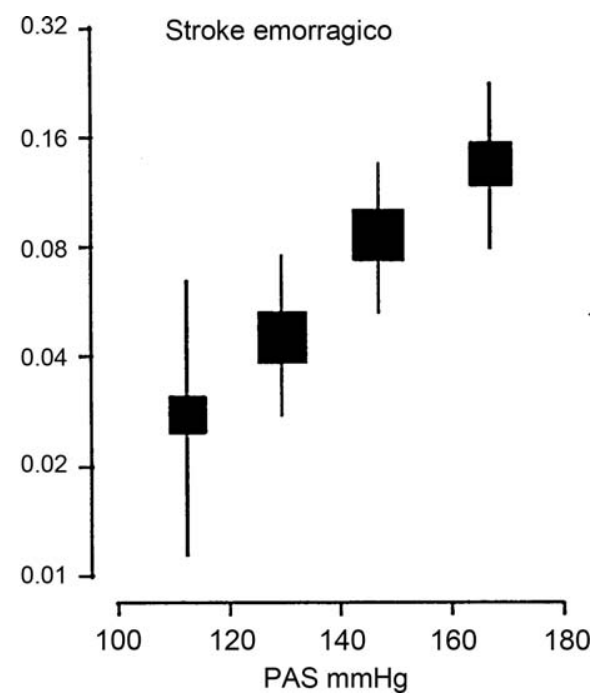

Figura 4 Incidenza dell'outcome primario stratificato secondo la PAS nello studio INVEST. Fonte: Messerli FH, et al. J Am Coll Cardiol 2009;54(20):1827-34.
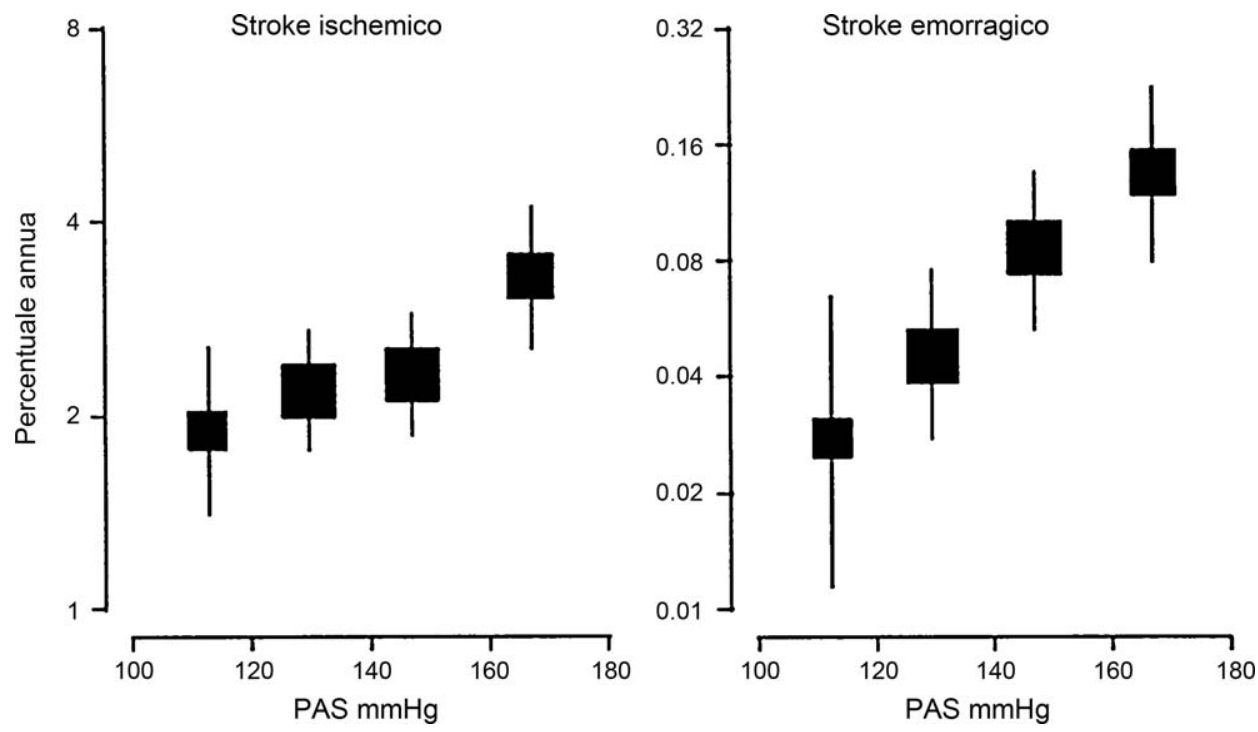

Figura 5 Studio PROGRESS: curva J non evidente per l'ictus ischemico $(p=0,0005)$ ed emorragico $(p<0,0001)$. Fonte: Arima H, et al. J Hypertens 2006;24(6):1201-8. 
Nel grande anziano i risultati sono controversi. Lo studio HYVET (Hypertension in the Very Elderly Trial) [42], nonostante una preanalisi in cui si evidenziava una riduzione dell'ictus nel gruppo in trattamento ma non della mortalità per tutte le cause, dopo 2 anni ha invece dimostrato che la terapia diminuisce anche la mortalità per tutte le cause. Occorre però considerare che il trial supporta solo il fatto che un target pressorio di $150 / 80 \mathrm{mmHg}$ sia ottimale, ma non fornisce una risposta circa l'utilità o, peggio, la pericolosità di ulteriori abbassamenti.

\section{Curva J e insufficienza renale}

Esiste una relazione lineare tra PAS e insufficienza renale. Nello studio IDNT (Irbesartan Diabetic Nephropathy Trial) [43] la riduzione della PAS, nei diabetici, a valori $<120 \mathrm{mmHg}$ ha portato a una minore mortalità CV e a una progressiva riduzione della proteinuria e del ricorso alla dialisi. Sempre nel diabetico, lo studio ADVANCE (Action in Diabetes and Vascular Disease - PreterAx and DiamicroN MRControlled Evaluation) [44] ha dimostrato che un paziente ogni 20 (IC 95\%: 15-30) assegnati al trattamento attivo eviterebbe l'insorgenza di un evento renale (prevalentemente lo sviluppo di una nuova albuminuria). Quindi, considerato che lo studio ADVANCE tratta anche diabetici con valori pressori di $140 / 90 \mathrm{mmHg}$, ridurre la pressione nei diabetici è una priorità assoluta, senza evidenza di una curva J.

Occorre però notare che, nelle popolazioni in emodialisi, una bassa PAS, anziché un'elevata PAS, è stata posta in relazione con la mortalità. Uno studio recente su 1.111 pazienti in emodialisi ha evidenziato un'associazione tra la bassa PAS nella predialisi e nella postdialisi e la mortalità nel breve termine (6 mesi); nel lungo termine non è stata individuata una relazione, né in pazienti con comorbilità CV né in pazienti senza tale comorbilità. Non è stata stabilita una relazione inversa con la PAD [45].

\section{Conclusioni}

Perché dunque questo titolo alla nostra rassegna? Che esista una curva J è scontato, poiché deve esistere una soglia sotto la quale una pressione troppo bassa non garantisce più la perfusione degli organi vitali; il punto, però, è riuscire a sapere quale sia questa soglia, anche perché, soprattutto negli ultimi trial di intervento, si è guardato più al rischio $\mathrm{CV}$ che alla sola pressione, per cui vi è stato, giustamente, un trattamento aggressivo della dislipidemia, del diabete e delle altre comorbilità. Tuttavia, bisogna essere molto prudenti nell'interpretare i dati forniti dai trial: l'associazione tra bassa PAD e rischio riscontrata in alcuni di essi non prova affatto che l'abbassamento della pressione ottenuto nella pratica clinica quotidiana aumenti la possibilità di eventi CV. Accettare acriticamente i risultati di questi trial potrebbe esporre milioni di persone a non essere trattate o a essere trattate insufficientemente.

Considerando anche la pressione di partenza, bisogna ricordare che i pazienti dei trial con una bassa PAD sono in genere più compromessi già di base, sia per patologie concomitanti (diabete, coronaropatia, malattie neoplastiche o sistemiche) sia per l'età. Quindi potrebbe non essere l'abbassamento della pressione ad aumentare il rischio, ma il fatto che fossero già pazienti ad alto rischio. Inoltre i pazienti con bassa PAD hanno anche, spesso, elevata PAS ed elevata PP (la cosiddetta ipertensione sistolica isolata, ISI) e le linee guida correnti invitano a ridurre la pressione dei pazienti con ISI. Ciò espone inevitabilmente a un ulteriore abbassamento della PAD e se il paziente è cardiopatico può essere pericoloso. È quanto sostengono molte analisi post hoc, sebbene alcuni autori contestino tali analisi e la metanalisi di Wang et al. [24] abbia dimostrato che i vantaggi si sono mantenuti con valori pressori $<70 \mathrm{mmHg}$ indipendentemente dall'età. Nei pazienti con CAD può essere discutibile, ma è questa la sfida: personalizzare la terapia antipertensiva.

In sostanza, quindi, non si tratta di discutere se esista una curva J, ma quale sia il punto $\mathrm{J}$, che comunque esiste, per la PAD e per la PAS per ogni paziente sottoposto a trattamento. In ogni caso l'ultimo aggiornamento delle linee guida europee, sulla base dei risultati dei maggiori trial del periodo 2007-2009, pur con le ovvie limitazioni delle analisi post hoc, vede una progressiva riduzione dell'incidenza degli eventi CV con il graduale abbassamento della PAS anche sotto i $120 \mathrm{mmHg}$ e della PAD anche sotto i $75 \mathrm{mmHg}$, nonostante i benefici addizionali di un'ulteriore riduzione della pressione siano piuttosto limitati: a questi bassi livelli di pressione arteriosa sono stati osservati benefici sul danno d'organo. Non è chiaro se al di sotto ci possa essere un fenomeno $\mathrm{J}$, eccetto, forse, nei pazienti con avanzata aterosclerosi. Sulla base dei dati correnti "può essere prudente abbassare la PAS/PAD nei range di 130-139/80-85 mmHg e possibilmente racchiudere $i$ valori più bassi in questo range" $[46,47]$.

Forse l'atteggiamento migliore è quello di non aspettare che pazienti a basso rischio diventino ad alto rischio, che a questo punto verrebbero esposti alle complicazioni di trattamenti difficili e talvolta, almeno in teoria, persino pericolosi.

In definitiva, come afferma Zanchetti, l'assioma non dovrebbe più essere "the lower the better", ma "the earlier the better" $[48,49]$.

\section{Conflitto di interesse}

Gli autori dichiarano di essere esenti da conflitto di interessi.

\section{Bibliografia}

[1] Farnett L, Mulrow CD, Linn WD, Lucey CR, Tuley MR. The J-curve phenomenon and the treatment of hypertension. Is there a point beyond which pressure reduction is dangerous? JAMA 1991;265(4):489-95.

[2] Hansson L. Antihypertensive treatment does the J-curve exist? Cardiovasc Drugs Ther 2000;14(4):367-72.

[3] Cruickshank JM. Antihypertensive treatment and the J-curve. Cardiovasc Drugs Ther 2000;14(4):373-9.

[4] Stewart IM. Relation of reduction in pressure to first myocardial infarction in patients receiving treatment for severe hypertension. Lancet 1979;1(8121):861-5.

[5] Cruickshank JM, Thorp JM, Zacharias FJ. Benefits and potential harm of lowering high blood pressure. Lancet 1987;1(8533): 581-4. 
[6] Leonetti G, Cuspidi C. Recent knowledge on the role of diastolic blood pressure in hypertensive patients on treatment. Recenti Prog Med 2008;99(4):191-9.

[7] Harrison DG, Barnes DH, Hiratzka LF, Eastham CL, Kerber RE, Marcus ML. The effect of cardiac hypertrophy on the coronary collateral circulation. Circulation 1985;71(6):1135-45.

[8] Owens P, O'Brien E. Hypotension in patients with coronary disease: can profound hypotensive events cause myocardial ischaemic events? Heart 1999;82(4):477-81.

[9] Merlo J, Ranstam J, Liedholm H, et al. Incidence of myocardial infarction in elderly men being treated with antihypertensive drugs: population based cohort study. BMJ 1996;313(7055): 457-61.

[10] Lewington S, Clarke R, Qizilbash N, Peto R, Collins R, Prospective Studies Collaboration. Age-specific relevance of usual blood pressure to vascular mortality: a meta-analysis of individual data for one million adults in 61 prospective studies. Lancet 2002;360(9349):1903-13.

[11] Pepine CJ, Handberg EM, Cooper-DeHoff RM, et al., INVEST Investigators. A calcium antagonist vs a non-calcium antagonist hypertension treatment strategy for patients with coronary artery disease. The International Verapamil-Trandolapril Study (INVEST): a randomized controlled trial. JAMA 2003;290(21): 2805-16.

[12] Messerli FH, Panjrath GS. The J-curve between blood pressure and coronary artery disease or essential hypertension: exactly how essential? J Am Coll Cardiol 2009;54(20):1827-34.

[13] Messerli FH, Mancia G, Conti CR, et al. Dogma disputed: can aggressively lowering blood pressure in hypertensive patients with coronary artery disease be dangerous? Ann Intern Med 2006;144(12):884-93.

[14] Williams B. Hypertension and the "J-curve". J Am Coll Cardiol 2009;54(20):1835-6.

[15] Tuomilehto J, Ryynänen OP, Koistinen A, Rastenyte D, Nissinen A, Puska P. Low diastolic blood pressure and mortality in a population-based cohort of 16913 hypertensive patients in North Karelia. Finland J Hypertens 1998;16(9):1235-42.

[16] SHEP Cooperative Research Group. Prevention of stroke by antihypertensive drug treatment in older persons with isolated systolic hypertension. Final results of the Systolic Hypertension in the Elderly Program (SHEP). JAMA 1991;265(24): 3255-64.

[17] Staessen JA, Fagard R, Thijs L, et al. Randomised double-blind comparison of placebo and active treatment for older patients with isolated systolic hypertension. The Systolic Hypertension in Europe (Syst-Eur) Trial Investigators. Lancet 1997;350(9080): 757-64.

[18] Bangalore S, Messerli FH, Wun CC, et al. J-curve revisited: An analysis of blood pressure and cardiovascular events in the Treating to New Targets (TNT) trial. Poster presented at the American Society of Hypertension 2009 Scientific Meeting. (San Francisco, CA; May 7, 2009).

[19] Amery A, Birkenhäger W, Brixko R, et al. Efficacy of antihypertensive drug treatment according to age, sex, blood pressure, and previous cardiovascular disease in patients over the age of 60. Lancet 1986;2(8507):589-92.

[20] Staessen J, Bulpitt C, Clement D, et al. Relation between mortality and treated blood pressure in elderly patients with hypertension: report of the European Working Party on High Blood Pressure in the Elderly. BMJ 1989;298(6687):1552-6.

[21] Williams B. The aorta and resistant hypertension. J Am Coll Cardiol 2009;53(5):452-4.

[22] Franklin SS, Larson MG, Khan SA, et al. Does the relation of blood pressure to coronary heart disease risk change with aging? The Framingham Heart Study. Circulation 2001;103(9): 1245-9.

[23] Bangalore S, Messerli FH, Franklin SS, Mancia G, Champion A, Pepine CJ. Pulse pressure and risk of cardiovascular outcomes in patients with hypertension and coronary artery disease: an
INternational VErapamil SR-trandolapril STudy (INVEST) analysis. Eur Heart J 2009;30(11):1395-401.

[24] Wang JG, Staessen JA, Franklin SS, Fagard R, Gueyffier F. Systolic and diastolic blood pressure lowering as determinants of cardiovascular outcome. Hypertension 2005;45(5):907-13.

[25] Hansson L, Zanchetti A, Carruthers SG, et al. Effects of intensive blood-pressure lowering and low-dose aspirin in patients with hypertension: principal results of the Hypertension Optimal Treatment (HOT) randomised trial. HOT Study Group. Lancet 1998;351(9118):1755-62.

[26] Psaty BM, Furberg CD, Kuller LH, et al. Association between blood pressure level and the risk of myocardial infarction, stroke, and total mortality: the Cardiovascular Health Study. Arch Intern Med 2001;161(9):1183-92.

[27] Hakala SM, Tilvis RS. Determinants and significance of declining blood pressure in old age. A prospective birth cohort study. Eur Heart J 1998;19(12):1872-8.

[28] Langer RD, Criqui MH, Barrett-Connor EL, Klauber MR, Ganiats TG. Blood pressure change and survival after age 75 . Hypertension 1993;22(4):551-9.

[29] Tervahauta M, Pekkanen J, Enlund H, Nissinen A. Change in blood pressure and 5-year risk of coronary heart disease among elderly men: the Finnish cohorts of the Seven Countries Study. J Hypertens 1994;12(10):1183-9.

[30] Satish S, Zhang DD, Goodwin JS. Clinical significance of falling blood pressure among older adults. J Clin Epidemiol 2001;54(9): 961-7.

[31] Taylor JO, Cornoni-Huntley J, Curb JD, et al. Blood pressure and mortality risk in the elderly. Am J Epidemiol 1991;134(5): 489-501.

[32] Boutitie F, Gueyffier F, Pocock S, Fagard R, Boissel JP, INDANA Project Steering Committee. INdividual Data ANalysis of Antihypertensive intervention. J-shaped relationship between blood pressure and mortality in hypertensive patients: new insights from a meta-analysis of individual-patient data. Ann Intern Med 2002;136(6):438-48.

[33] Greenberg JA. Removing confounders from the relationship between mortality risk and systolic blood pressure at low and moderately increased systolic blood pressure. J Hypertens 2003;21(1):49-56.

[34] Vaccarino V, Holford TR, Krumholz HM. Pulse pressure and risk for myocardial infarction and heart failure in the elderly. J Am Coll Cardiol 2000;36(1):130-8.

[35] Somes GW, Pahor M, Shorr RI, Cushman WC, Applegate WB. The role of diastolic blood pressure when treating isolated systolic hypertension. Arch Intern Med 1999;159(17):2004-9.

[36] Benetos A, Thomas F, Safar ME, Bean KE, Guize L. Should diastolic and systolic blood pressure be considered for cardiovascular risk evaluation: a study in middle-aged men and women. J Am Coll Cardiol 2001;37(1):163-8.

[37] Glynn RJ, Chae CU, Guralnik JM, Taylor JO, Hennekens CH. Pulse pressure and mortality in older people. Arch Intern Med 2000;160(18):2765-72.

[38] Kannel WB, Wilson PW, Nam BH, D’Agostino RB, Li J. A likely explanation for the J-curve of blood pressure cardiovascular risk. Am J Cardiol 2004;94(3):380-4.

[39] Blacher J, Staessen JA, Girerd X, et al. Pulse pressure not mean pressure determines cardiovascular risk in older hypertensive patients. Arch Intern Med 2000;160(8):1085-9.

[40] Sleight P, Redon J, Verdecchia P, et al., ONTARGET investigators. Prognostic value of blood pressure in patients with high vascular risk in the Ongoing Telmisartan Alone and in combination with Ramipril Global Endpoint Trial study. J Hypertens 2009;27(7):1360-9.

[41] Arima H, Chalmers J, Woodward M, et al., PROGRESS Collaborative Group. Lower target blood pressures are safe and effective for the prevention of recurrent stroke: the PROGRESS trial. J Hypertens 2006;24(6):1201-8. 
[42] Beckett NS, Peters R, Fletcher AE, et al., HYVET Study Group. Treatment of hypertension in patients 80 years of age or older. $\mathrm{N}$ Engl J Med 2008;358(18):1887-98.

[43] Pohl MA, Blumenthal S, Cordonnier DJ, et al. Independent and additive impact of blood pressure control and angiotensin II receptor blockade on renal outcomes in the Irbesartan Diabetic Nephropathy Trial: clinical implications and limitations. J Am Soc Nephrol 2005;16(10):3027-37.

[44] Patel A, MacMahon S, Chalmers J, et al., ADVANCE Collaborative Group. Effects of a fixed combination of perindopril and indapamide on macrovascular and microvascular outcomes in patients with type 2 diabetes mellitus (the ADVANCE trial): a randomised controlled trial. Lancet 2007;370(9590): 829-40.

[45] Bos WJ, van Manen JG, Noordzij M, Boeschoten EW, Krediet RT, Dekker FW. Is the inverse relation between blood pressure and mortality normalized in "low-risk" dialysis patients? J Hypertens 2010;28(3):439-45.

[46] Mancia G, Laurent S, Agabiti-Rosei E, et al., European Society of Hypertension. Reappraisal of European guidelines on hypertension management: a European Society of Hypertension Task Force document. J Hypertens 2009;27(11):2121-58.

[47] Grassi G, Quarti-Trevano F, Dell'Oro R, Mancia G. The "J curve" problem revisited: old and new findings. Curr Hypertens Rep 2010;12(4):290-5.

[48] Zanchetti A, Grassi G, Mancia G. When should antihypertensive drug treatment be initiated and to what levels should systolic blood pressure be lowered? A critical reappraisal. J Hypertens 2009;27(5):923-34.

[49] Zanchetti A. Bottom blood pressure or bottom cardiovascular risk? How far can cardiovascular risk be reduced? J Hypertens 2009;27(8):1509-20. 